\title{
CPL207280, a Novel G Protein-Coupled Receptor 40/Free Fatty Acid Receptor 1-Specific Agonist, Shows a Favorable Safety Profile and Exerts Antidiabetic Effects in Type 2 Diabetic Animals
}

\author{
Katarzyna Bazydlo-Guzenda, Pawel Buda, Mikolaj Matloka, Mateusz Mach, Filip Stelmach, \\ Radoslaw Dzida, Damian Smuga, Joanna Hucz-Kalitowska, Malgorzata Teska-Kaminska, \\ Varvara Vialichka, Krzysztof Dubiel, Bozena Kaminska, Maciej Wieczorek, and \\ Jerzy Pieczykolan
}

Research and Development Centre, Celon Pharma SA, Kazun Nowy, Poland (K.B.-G., P.B., Mi.M., Ma.M., F.S., R.D., D.S., J.H.-K., M.T.-K., V.V., K.D., M.W., J.P.), and Postgraduate School of Molecular Medicine, Medical University of Warsaw, Warsaw, Poland (K.B.-G., B.K.).

Received February 18, 2021; accepted July 14, 2021

\section{ABSTRACT}

G protein-coupled receptor (GPR) 40 is a free fatty acid receptor mainly expressed in pancreatic $\beta$-cells activated by medium- and long-chain fatty acids and regulating insulin secretion via an increase in cytosolic free calcium $\left(\left[\mathrm{Ca}^{2+}\right]_{\mathrm{i}}\right)$. Activation of GPR40 in pancreatic $\beta$-cells may improve glycemic control in type 2 diabetes through enhancement of glucose-stimulated insulin secretion. However, the most clinically advanced GPR40 agonist-TAK-875 (fasiglifam) - was withdrawn from phase III because of its hepatotoxicity resulting from the inhibition of pivotal bile acid transporters. Here, we present a new, potent CPL207280 agonist and compare it with fasiglifam in numerous in vitro and in vivo studies. CPL207280 showed greater potency than fasiglifam in a $\mathrm{Ca}^{2+}$ influx assay with a human GPR40 protein $\left(\mathrm{EC}_{50}=80 \mathrm{vs}\right.$. $270 \mathrm{nM}$, respectively). At the $10 \mu \mathrm{M}$ concentration, it showed 3.9 times greater enhancement of glucose-stimulated insulin secretion in mouse MIN6 pancreatic $\beta$-cells. In Wistar Han rats and C57BL6 mice challenged with glucose, CPL207280 stimulated 2.5 times greater insulin secretion without causing hypoglycemia at $10 \mathrm{mg} / \mathrm{kg}$ compared with fasiglifam. In three diabetic rat models, CPL207280 improved glucose tolerance and increased insulin area under the curve by $212 \%, 142 \%$, and $347 \%$, respectively. Evaluation of potential off-target activity (Safety47) and selectivity of CPL207280 (at $10 \mu \mathrm{M}$ ) did not show any significant off-target activity. We conclude that CPL207280 is a potent enhancer of glucose-stimulated insulin secretion in animal disease models with no risk of hypoglycemia at therapeutic doses. Therefore, we propose the CPL207280 compound as a compelling candidate for type 2 diabetes treatment.

\section{SIGNIFICANCE STATEMENT}

GPR40 is a well-known and promising target for diabetes. This study is the first to show the safety and effects of CPL207280, a novel GPR40/free fatty acid receptor 1 agonist, on glucose homeostasis both in vitro and in vivo in different diabetic animal models. Therefore, we propose the CPL207280 compound as a novel, glucose-lowering agent, overcoming the unmet medical needs of patients with type 2 diabetes.
The study was financed by Celon Pharma SA, the National Center of Research and Development [POIR.01.01.01.-00-0334/17], and the Ministry of Science and Higher Education [50/DW/2017/01/1]

K.B.-G., P.B., Mi.M., Ma.M., F.S., D.S., J.H.-K., M.T.-K., V.V., K.D , and J.P. are employees at Celon Pharma SA; R.D. at the time of his direct involvement in the project was the full-time employee of Celon Pharma SA; M.W. is the owner and CEO of Celon Pharma SA; Mi.M., M.T.-K., K.D., J.P., and M.W. hold stocks of Celon Pharma SA.

https://doi.org/10.1124/molpharm.121.000260.

\section{Introduction}

G protein-coupled receptor (GPR) 40 (or FFA1) is a free fatty acid receptor mainly expressed in pancreatic $\beta$-cells (Itoh and Hinuma, 2005; Schnell et al., 2007). It mediates signaling from free fatty acid (FFA) to modulate glucose-stimulated insulin secretion (GSIS) (Briscoe et al., 2003; Itoh et al., 2003). GPR40 emerged as an antidiabetic target and was proposed as a novel treatment modality for patients with type 2

ABBREVIATIONS: ADR, adverse drug reaction; AUC, area under the curve; BSA, bovine serum albumin; F, bioavailability; FFA, free fatty acid; FFA1, free fatty acid receptor 1; FFA2, free fatty acid receptor 2; FFA3, free fatty acid receptor 3; FFAF, free fatty acids free; GK, GotoKakizaki rat; GPCR, G protein-coupled receptor; GPR, G protein-coupled receptor; GSIS, glucose-stimulated insulin secretion; GTT, glucose tolerance test; HBSS, Hanks' balanced salt solution; hERG, the human ether-a-go-go related gene; HG, high glucose; KRBH, Krebs-Ringer bicarbonate HEPES buffer; LG, low glucose; PEG, polyethylene glycol; PPAR, proliferator-activated receptor; SU, sulfonylurea; T2D, type 2 diabetes; ZDF, Zucker diabetic fatty rat; ZDSD, Zucker diabetic Sprague-Dawley rat. 
diabetes (Hara et al., 2011) in whom blood glucose control by popular drugs (i.e., metformin) is insufficient. Modulation of GSIS by FFA is perceived as an adaptive mechanism in a T2D milieu. A high concentration of lipids aggravates insulin resistance while enhancing insulin secretion to match the systemic demand (Nagasumi et al., 2009). Glucose dependence of the GPR40 insulinotropic activity is particularly important from the clinical perspective. Interestingly, it may allow prevention of the main adverse effect (iatrogenic hypoglycemia) of the currently popular sulfonylureas (SUs), which stimulates insulin secretion irrespective of glucose levels (Sola et al., 2015). Consequently, sulfonylureas impart a constant workload on $\beta$-cells instead of ensuring a fine-tuning of insulin secretion depending on the demand. This, in turn, leads to a $\mathrm{Ca}^{2+}$ overload and propels $\beta$-cell dysfunction, leading to cell death (Iwakura et al., 2000).

Impairments of $\beta$-cells function and mass decline as the T2D signs of progress are caused in part by elevated FFAs described as glucolipotoxicity (Poitout et al., 2010). Although GPR40 is a receptor for FFAs that might suggest its deleterious effects, aggregating evidence shows that GPR40 is not the gateway for glucolipotoxicity (Latour et al., 2007; Kebede et al., 2008; Lan et al., 2008; Alquier and Poitout, 2009). This fundamental feature permits GPR40 to become a promising candidate target for a novel secretagogue. Nevertheless, for successful therapy, the novel GPR40 agonist must also be safe, which means being structurally different from FFAs, to circumvent their toxicity. Moreover, to become fully developed, the drug must show much greater potency than FFAs and must not induce tachyphylaxis (Sharma and Alonso, 2014). Meeting all of these three conditions has become particularly challenging on the pathway of GPR40 synthetic agonist development. For example, fasiglifam (TAK-875; Takeda) was shown not to cause tachyphylaxis, and it demonstrated 400 times greater potency at GPR40 than the cognate ligand oleic acid (Tsujihata et al., 2011; Ito et al., 2016). It showed an antidiabetic effect in numerous animal diabetes models by improving glucose control in both acute and chronic administration (fasting parameters) (Ito et al., 2013, 2016). More importantly, it improved glucose tolerance and hemoglobin HbA1c levels in a small phase II clinical study $(n>50)$ in a cohort of mixedethnicity subjects (Burant et al., 2012). However, in a largepopulation phase III study ( $n>5000)$, it demonstrated safety alerts in $2.5 \%$ of individuals (Marcinak et al., 2017). Although its structure was profoundly different to that of FFA, it turned out to be toxic for hepatocytes in the liver (especially through bile acid inhibition) (Li et al., 2015; Wolenski et al., 2017). Ever since TAK-875 entered phase III clinical trials, we have kept it as a benchmark for comparison purposes.

We have developed CPL207280, a new agonist of GPR40, which is at least three times more potent at human GPR40 and 10 times less toxic on insulinoma cells than TAK-875, which translates into a broader therapeutic window. Here, we compare the biologic properties of CPL207280 and TAK-875, and we have found that the addition of CPL207280 improved glucose tolerance in MIN6 pancreatic $\beta$-cells as well as in healthy Wistar Han rats and diabetes rat models (GK, ZDSD, $\mathrm{ZDF}$ rats). Incubation of CPL207280 with the rat and mouse insulinoma (INS-1 and MIN6) cells for 48 hours did not cause significant cell viability changes, whereas TAK- 875 reduced rat and mouse insulinoma cell viability at higher concentrations, indicative of a narrower safety window.

\section{Materials and Methods}

Cells and Compounds. CHO cell line overexpressing human GPR40 receptor and aequorin was obtained from Perkin Elmer (ES652-AF). Mouse insulinoma (MIN6) (C0018008) and rat insulinoma (INS-1) cell lines (C0018007) were obtained from AddexBio. GW1100 was obtained from Calbiochem (371830). Cells were routinely tested for possible mycoplasma contamination.

CPL207280 and TAK-875 molecules were synthesized in-house with a purity $>98 \%$ as based on high-performance liquid chromatography (HPLC) area integration with UV detection at a wavelength of $254 \mathrm{nM}$ (Mach, et al., 2019).

Aequorin Assay. CHO cells overexpressing human GPR40 receptor and aequorin were seeded in a T25 flask with a Ham's F-12 full medium 24 hours before the experiment. Subsequently, $2.5 \times 10^{6}$ cells were collected by trypsinization with $0.25 \%$ trypsin for 3 minutes, centrifuged (200g, 5 minutes), and resuspended in a $1-\mathrm{ml}$ HBSS buffer (Gibco). Free fatty acids free (FFAF) 10\%-BSA (final concentration $0.015 \%$ ) and Coelenterazine $\mathrm{h}$ (in a methanol solution, final concentration $5 \mu \mathrm{M}$ ) were diluted in an HBSS buffer, added to the cell suspension, and agitated gently for 2 hours at room temperature (RT). Next, cells were suspended with HBSS and mixed for the next hour. Then, cells were transferred into a glass cylinder with a magnetic mixer, mixed, and seeded (final density 5000 cells per well) on a 96-well plate comprising an array of investigated compounds in dilutions. The measurement of the luminescence signal was carried out continuously for 1 minute, and the resulting curves were used to calculate the area under the curve (AUC). CPL207280 activity was calculated as a percentage of $\alpha$-linolenic acid activity (used as a control) at $10 \mu \mathrm{M}$.

Cell Viability Assay. For the cell viability assay, $20 \times 10^{3}$ INS-1 or $50 \times 10^{3}$ MIN6 cells were seeded in $100 \mu \mathrm{l}$ of RPMI 1640 or Dulbecco's modified Eagle's medium full media (with $0.05 \mathrm{mM} \beta$-mercaptoethanol) in a 96-well plate (transparent bottoms) coated with poly(D-lysine) (Corning). On the following day, $100 \mu \mathrm{l}$ of the full media containing $500 \times$ RT-Glo (Promega) and a $2 \times$ concentrated tested compound at 5 concentrations up to $100 \mu \mathrm{M}$ was added. After 48 hours of incubation at $37^{\circ} \mathrm{C}$ and $5 \% \mathrm{CO}_{2}$, the luminescence was measured using the VICTOR Nivo Multimode Microplate Reader (Perkin Elmer).

Insulin Secretion Assay. MIN6 or INS-1E cells $\left(50 \times 10^{3}\right.$ per well) were seeded in a 96 -well plate (transparent bottoms) coated with poly(D-lysine). After 72 or 48 hours, respectively, cells were washed twice with Krebs-Ringer bicarbonate HEPES buffer (KRBH) (containing $2.8 \mathrm{mM}$ glucose and $0.1 \%$ FFAF BSA) and incubated in this buffer for 2 hours at $37^{\circ} \mathrm{C}$. Before the end of starvation (15 minutes), GW1100 was added to dedicated wells with INS-1E cells to a final concentration of $10 \mu \mathrm{M}$. Next the medium was replaced with KRBH supplemented with $20 \mathrm{mM}$ glucose and CPL207280 and TAK-875 at concentrations up to $40 \mu \mathrm{M}$ or GW1100 at a concentration of $10 \mu \mathrm{M}$, and cells were incubated for 1 hour. Finally, $100 \mu \mathrm{l}$ of supernatant was collected, centrifuged at $1500 \mathrm{~g}$ for 5 minutes, and frozen at $-80^{\circ} \mathrm{C}$. On the next day, the samples were diluted in $\mathrm{KRBH}$, and insulin levels were determined using a mouse or rat insulin ELISA kit (Mercodia) in line with the manufacturer's instructions. Cells remaining in the well were lysed with a radioimmunoprecipitation assay (RIPA) buffer, and the total protein concentration, used for insulin concentration normalization, was measured using a BCA kit (Thermo Fisher).

Off-Target Analysis. The selectivity of CPL207280 against representative members of GPCR and PPAR was performed using GPCR Screening and Profiling Services by DiscoverX (Eurofins DiscoverX Corporation, Fremont, CA) in line with the service provider's protocol. The assays were performed at $10 \mu \mathrm{M}$, utilizing the Calcium No Wash ${ }^{\text {PLUS }}$ (FFAR2), Path-Hunter $\beta$-Arrestin (FFAR3, GPR55, GPR119, GPR120, GPR142), Hit Hunter cAMP assay (glucagon-like peptide-1 receptor (GLP1R), and NHR Protein Interaction (PPAR $\alpha$, PPAR $\gamma$ ) functional cell-based assays. The compound activity was analyzed using the chemical and biological information system (CBIS) data analysis suite (ChemInnovation, CA). 
Safety 47 assay, a panel of 47 potential off-target proteins, was performed by DiscoverX (Eurofins DiscoverX Corporation, Fremont, CA) in line with the service provider's protocol. The screening was performed at $10 \mu \mathrm{M}$ using functional cell-based, binding, and enzymatic assays utilizing the Calcium No Wash ${ }^{\text {PLUS }}$ assay (adenosine A2A receptor -ADORA2A, ADRA1A, arginine vasopressin receptor 1A - AVPR1A, cholecystokinin A receptor -CCKAR, CHRM1, CHRM3, endothelin receptor type A -EDNRA, HRH1, HTR2A, HTR2B), NHR Protein Interaction assay glucocorticoid receptor -(GR), Nuclear Translocation assay androgen receptor -(AR), Ion Chanel Assay [calcium channel, voltage-dependent, L type, alpha 1C subunit -CAV1.2, $\gamma$-aminobutyric acid type A receptor -GABAA, hERG, HTR3A, potassium voltage-gated channel KQT-like member 1/minimal potassium channel -KvLQT1/mink, nicotinic acetylcholine receptors -nAChR(a4/b2), voltage-gated sodium channel subunit $\alpha$-NAV1.5, N-methyl-D-aspartate receptor -NMDAR (1A/ 2B), DAT], Hit Hunter cAMP assay (HTR1B, OPRD1, OPRK1, ADRA2A, ADRB1, ADRB2, CHRM2, CNR1, CNR2, DRD1, DRD2S, HRH2, HTR1A), KINOMEscan kinase binding assays (insulin receptor -INSR, LCK, ROCK1, VEGFR2), Neurotransmitter Transporter Uptake Assay (DAT, norepinephrine transporter -NET, serotonin transporter -SERT), and a variety of enzyme inhibition assays (acetylcholinesterase - AChE, COX1, COX2, monoamine oxidase A - MAOA, PDE3A, PDE4D2).

The compound activity was analyzed using the chemical and biological information system (CBIS) data analysis suite (ChemInnovation, CA). Detailed information about each assay condition is available at the Eurofins DiscoverX Corporation website.

Animal Care. The following animals were used this study: 8- to 9-week-old male C57BL6/cmdb mice (Experimental Center of the Medical University in Bialystok), 8- to 10-week-old male Wistar Han rats [Crl:WI (Han); Charles River], 10- to 12-week-old male ZDSD rats (ZDSD/Pco; Crown Bioscience), 10- to 12-week-old GK rats (GK/ MolTac; Taconic), and 9-week-old ZDF and ZL rats (Vital River Laboratories Research Models and Services; Beijing, China). All animals (except ZDF rats) were maintained on a standard chow diet (recommended by the supplier) and acclimated to the experimental facility for a minimum of 7 days before treatment. The ZDF rats were fed with a diet of Purina Rodent LabDiet 5C08 (equivalent to LabDiet 5008) for the first week upon arrival. Next, all rats were put on a diet regimen in which the animals had access to food for 8 hours during the day and were fasted overnight.

All animals were housed in cages on a ventilated rack. The animals were housed under a 12-hour light/dark cycle (and were allowed ad libitum access to water). During quarantine, animals were marked, weighed, and had a blood glucose measurement (adapted to venous punctures in the tail vein and the experimenter performing the measurements). The fasting blood glucose level was used for animal randomization to experimental groups $(n=7 / 8)$ in the glucose tolerance test (GTT) study.

All procedures on animals were conducted in accordance with the relevant national regulatory guidelines, and individual experiments were approved by the Local Bioethics Committee in Poland (approval number: 56/2013; 32/2016; 42/2017).

Pharmacokinetic Study. Wistar Han rats $(n=4 / 5)$ were fasted for 12 hours, and at specific times after oral or intravenous administration of CPL207280 (5\% DMSO/40\% PEG300/55\% PBS) $(0.08,0.25$, $0.5,1,2,4,7,12$, and 24 hours), each animal was bled by venipuncture, and the samples were collected into tubes containing the anticoagulant, $\mathrm{K}_{2}$ EDTA. Tubes were placed on wet ice pending processing. Next, the samples were centrifuged ( $1200 \mathrm{~g}$ for 5 minutes at approximately $4^{\circ} \mathrm{C}$ ). The analyte (CPL207208) was extracted from an aliquot of the animal $\mathrm{K}_{2}$ EDTA plasma in line with the protein precipitation assay. The extracted samples were injected into an ultrahigh-performance liquid chromatography (Infinity II Agilent Technologies 1290) coupled with tandem mass spectrometry (Sciex QTrap 6500). The calibration range for this assay spanned from 10.0 to $2000.0 \mathrm{ng} / \mathrm{ml}$. The mean $\mathrm{C}_{\max }$ and $\mathrm{T}_{\max }$ values from Table 3 are read from a compound concentration plot.
Glucose Tolerance Test. Male C57BL6/cmdb mice were fasted for 12 hours and administered intravenously (tail vein) with the $10 \mathrm{mg} /$ $\mathrm{kg}$ of CPL207280, TAK-875, or vehicle (5\% DMSO/40\% PEG300/55\% PBS); the glucose bolus was administered immediately after that. Just before and after $0.25,0.5,1,2,2.5$, and 3 hours after administration of a compound, glucose concentration in blood was measured.

Wistar Han rats were fasted for 12 hours and then administered orally with $10 \mathrm{mg} / \mathrm{kg}$ of CPL207280, TAK-875, or the vehicle $(5 \%$ DMSO/40\% PEG300/55\% PBS), and the first glucose bolus was administered immediately after that. The second glucose bolus was administered 6 hours later. Prior to the glucose administration and after $0.08,0.25,0.5,1,2,3,6.25,6.5,7,8$, and 9 hours, glucose concentration in blood was measured. Insulin concentration in blood was measured only during the first glucose bolus administration.

ZDSD rats were starved for 6 hours, after which they were orally administered CPL207280 at 3, 10, 30, and $100 \mathrm{mg} / \mathrm{kg}$ b.wt.; TAK-875 at $100 \mathrm{mg} / \mathrm{kg}$; glibenclamide at $30 \mathrm{mg} / \mathrm{kg}$; or the vehicle $(0.9 \% \mathrm{NaCl})$. At 1 hour later, a glucose bolus was administered. Blood glucose and insulin were measured 1 and 0.5 hours prior to glucose administration, as well as during the glucose challenge for up to 4 hours.

GK rats were fasted for 6 hours and next given orally $30 \mathrm{mg} / \mathrm{kg}$ CPL207280, TAK-875, or the vehicle (5\% DMSO/55\% PEG300/40\% PBS). At 1 hour later, animals were challenged with glucose. Blood glucose and insulin were measured right before drug and glucose administration and over the course of the GTT at $t=0.08,0.25,0.5$, $1,2,3$, and 6 hours. Because of the high concentration of fasting blood glucose observed in ZDSD and GK rats, there was a concern about the physical condition of the animals during the study after being fasted overnight. Therefore, in line with the animal supplier's suggestion, we decided to reduce the fasting time from 12 to 6 hours. This was sufficient for obtaining glucose level standardization and did not affect the animals' condition or the experiment results.

ZDF rats were fasted overnight for 16 hours and orally gavaged at 5 and $15 \mathrm{mg} / \mathrm{kg}$ of CPL207280, $15 \mathrm{mg} / \mathrm{kg}$ of TAK-875, or the vehicle $(0.9 \% \mathrm{NaCl})$, followed by an oral glucose bolus 0.5 hours after the dose. Blood samples for glucose and insulin measurement were collected from each animal right before drug administration; just before glucose loading; and after $0.25,0.5,1$, and 2 hours after glucose administration. In all in vivo studies, animals (except ZDF rats) were intraperitoneally challenged with a glucose bolus $(2 \mathrm{~g} / \mathrm{kg})$. At different time points, one or two drops of blood were directly used for a glucose measurement using a standard Accu-ChekPerforma glucometer. For insulin measurement, blood was sampled from the jugular vein, allowed to clot for a minimum of 30 minutes at room temperature, and then centrifuged at $4^{\circ} \mathrm{C}, 10,000 \mathrm{rpm}$, for 10 minutes. The resultant serum was analyzed using a Rat Insulin ELISA kit (Mercodia).

The time and administration mode of glucose during the GTT study were changed during the compound development. In the first, proof-ofconcept animal studies on healthy animals, glucose was administrated intravenously immediately after compound administration because of CPL207280's good pharmacokinetic properties (Table 3). During the compound development, to allow for a comparison of CPL207280 results with other GPR40 agonists, whose pharmacokinetics are different from ours and the $\mathrm{T}_{\max }$ occurs later e.g. TAK-875 (Negoro et al., 2010), the time of glucose bolus administration. During the GTT study in ZDSD and GK diabetic rat models, we observed that insulin concentration peak occurred 0.5 hours after compound administration. After combining those findings with the data from the TAK-875 study on ZDF rats (Ito et al., 2013) in which glucose was administered orally, we decided to adapt this study to the literature data.

Statistical Analysis. Statistical analysis was performed using GraphPad Prism software (version 7). All experiments presented in this manuscript were exploratory, so the null hypothesis has not been stated. Experimental data are presented as means \pm S.D. EC $_{50}$ value was determined based on logarithm values using nonlinear regression (curve fit) and four-parameter variable slope equation. Statistical significance between the mean of the treated groups was compared with the mean of the control group using one-way or two-way ANOVA followed by a post 

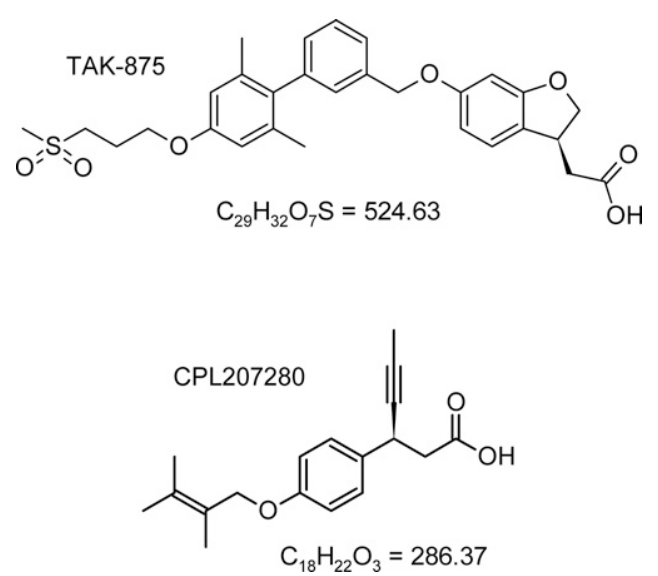

Fig. 1. Chemical structure of (3S)-3-\{4-[(2,3-dimethylbut-2-en-1-yl)oxy]phenyl\}hex-4-ynoic acid-CPL207280 and TAK-875.

hoc comparison (Dunnet or Sidak). A $P$ value $<0.05$ was considered statistically significant. Dose dependence between the doses was analyzed using one-way ANOVA, followed by a post hoc comparison (Tukey's).

\section{Results}

\section{Characteristics of CPL207280 Activity In Vitro}

After reviewing the compound library in search of GPR40 agonists, we identified CPL207280 (Fig. 1) as the lead structure for further development. In contrast to TAK-785 and other GPR40 agonists, CPL207280, by incorporation into its structure small acyclic motifs, has significantly reduced the molecular weight and overall lipophilicity at physiologic $\mathrm{pH}=$ 7.4 to avoid potential liver toxicity adverse effects (Mach et al., 2019).

The activity of CPL207280 on GPR40 was characterized using a variety of biochemical and cell-based assays on cell lines that expressed GPR40. To compare the efficacy of CPL207280 and TAK-875 (the best characterized GPR40 agonist so far), we used the $\mathrm{Ca}^{2+}$ release assay in CHO cells expressing a human GPR40. In this assay, $\mathrm{Ca}^{2+}$ released from internal stores of endoplasmic reticulum (ER) competes

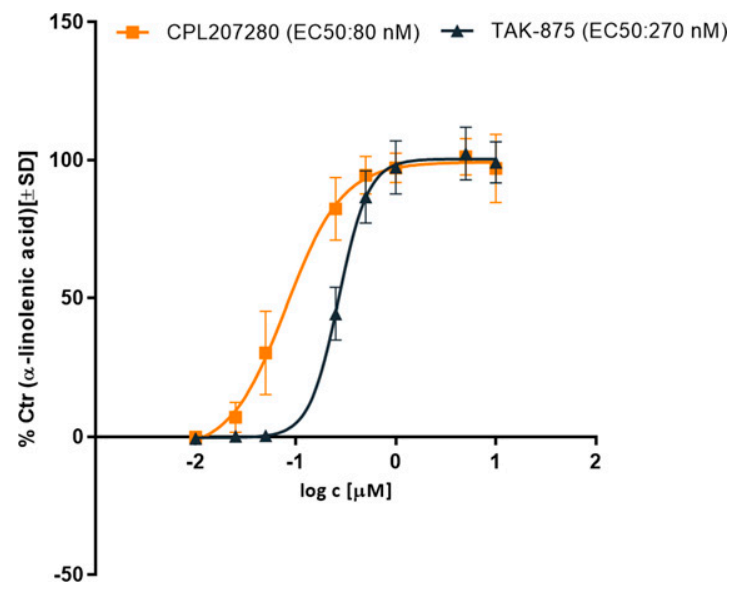

Fig. 2. $\mathrm{EC}_{50}$ values of CPL207280 in $\mathrm{Ca}^{2+}$ secretion assay on $\mathrm{CHO}$ cells overexpressing human GPR40. The activity of CPL207280 and TAK-875 is expressed as a percentage of the activity of the naturally occurring GPR40/FFA1 ligand $\alpha$-linolenic acid in aequorin $\mathrm{Ca}^{2+}$ flux. Data are presented as means \pm S.D. from three independent experiments performed in two technical replicates each. with coelenterazine in a complex with aequorin, resulting in a shift of absorbance at $470 \mathrm{~nm}$. The measurements were performed in the presence of a low concentration of FFAF BSA to minimize the effect of albumin binding. CPL207280 demonstrated $\mathrm{EC}_{50}$ of $80 \mathrm{nM}$ and TAK- 875 of $270 \mathrm{nM}$ (Fig. 2 ), which shows three times lesser potency of TAK- 875 . CPL207280 tested with the use of the Calcium No Wash ${ }^{\text {PLUS }}$ Assay, Path-Hunter $\beta$-Arrestin, and NHR Protein Interaction assays did not demonstrate any activity on the related GPR receptors, i.e., GPR41 (FFA2), GPR43 (FFA3), GPR55, GPR119, GPR120, GPR142, PPAR $\alpha$, or PPAR $\gamma$ when applied at $10 \mu \mathrm{M}$ concentration; this indicates that the activity was specific to GPR40 (Table 1).

\section{Potentiation of Insulin Secretion by CPL207280 in Mouse and Rat Insulinoma Cells}

A GPR40 receptor is expressed predominantly in $\beta$-cells of the pancreatic islet, and its activation improves glucose-stimulated insulin secretion (Itoh and Hinuma, 2005; Schnell et al., 2007). We studied the insulinotropic effect of CPL207280 and TAK-875 in mouse (MIN6) and rat (INS-1E) insulinoma cells. Cells were maintained for 2 hours under low-glucose (LG) $(2.8 \mathrm{mM})$ conditions and next were exposed to high glucose (HG) $(20 \mathrm{mM})$ for 1 hour. The addition of compounds to HG cultures increased the amount of secreted insulin both in MIN6 (Fig. 3A) and INS-1E (Fig. 3B) cells. A significant effect for TAK-875 was observed only at the highest concentration $(40 \mu \mathrm{M})$, whereas for CPL207280, a similar effect was present at all tested concentrations, suggesting greater potency of the latter (Fig. 3A). The effect for CPL207280 was dose-independent. To study whether CPL207280s effect is mediated via the GPR40 receptor, we treated the INS-1E cells with a selective antagonist of the GPR40 receptor, GW1100. In the presence of GW1100, the glucose-stimulated insulin secretion by CPL207280 was abolished (Fig. 3B). The fact that GW1100 blocked this action confirmed that the observed GSIS effect of CPL207280 is mediated via GPR40.

\section{CPL207280 Treatment Does Not Induce Cytotoxicity In Vitro}

Calcium overload in pancreatic $\beta$-cells compromises their viability through a mechanism known as excitotoxicity (Maedler et al., 2005). This phenomenon is ascribed to SUs as the adverse effect contributing to its secondary failure. Sulfonylureas hold the plasma membrane in the depolarized state by inhibition of $\mathrm{K}_{\mathrm{ATP}}$. This in turn causes a voltage-gated calcium channel-dependent influx and excess of $\mathrm{Ca}^{2+}$ in the cytosol. The activation of GPR40 also leads to rises in $\left[\mathrm{Ca}^{2+}\right]_{\mathrm{i}}$ and hence may aggravate excitotoxicity. Furthermore, GPR40 was found to activate voltage-gated calcium channels, which inevitably contributes to elevated intracellular $\mathrm{Ca}^{2+}$ levels (Fujiwara et al., 2005). To address potential excitotoxicity, we tested whether long-term treatment with CPL207280 may reduce the viability of $\beta$-cells under stimulatory conditions. INS-1 and MIN6 cells were incubated in media supplemented with $11.2 \mathrm{mM}$ glucose with the tested agonists at various concentrations for 48 hours. Next, cell viability was measured with an RT-Glo assay, which is based on luciferase activity in the presence of intact cellular metabolism. CPL207280 did not affect the viability of MIN6 cells at concentrations up to $100 \mu \mathrm{M}$. By contrast, TAK- 875 reduced 
TABLE 1

Selectivity study results

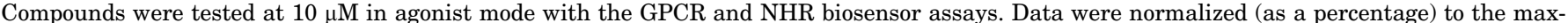
imal and minimal response observed in the presence of control ligand and vehicle. For Gi cAMP assays, $15 \mu \mathrm{M}$ forskolin was used.

\begin{tabular}{|c|c|c|c|c|c|c|c|c|c|}
\hline Activity [\% Ctr] & FFAR2 & FFAR3 & GPR119 & GPR120 & GPR55 & GPR142 & GLP1R & $\operatorname{PPAR} \gamma$ & $\operatorname{PPAR} \alpha$ \\
\hline Assay & Calcium flux & cAMP & $\beta$-Arrestin & $\beta$-Arrestin & $\beta$-Arrestin & $\beta$-Arrestin & $\beta$-Arrestin & $\begin{array}{l}\text { NHR Protein } \\
\text { Interaction }\end{array}$ & $\begin{array}{l}\text { NHR Protein } \\
\text { Interaction }\end{array}$ \\
\hline CPL207280 & -1 & 28.5 & 9.1 & 0.5 & 6.3 & 8.5 & -2.6 & 1.2 & 0.9 \\
\hline TAK-875 & 9.1 & 22.8 & 7.3 & 6 & 14.8 & -13.4 & -1.5 & 4.1 & 8.7 \\
\hline
\end{tabular}

cell viability by $63 \%$ at higher concentrations (Fig. $4 \mathrm{~A})$. A considerably stronger effect occurs in INS-1 cells, for which reduction of cell viability by $25 \%$ was observed after treatment with CPL207280 only at $100 \mu \mathrm{M}$. In case of TAK-875, $37 \%$ less viability was detected at $5 \mu \mathrm{M}$ with up to $100 \%$ of dead cells at $10 \mu \mathrm{M}$ (Fig. 4B).

Safety-related drug failures remain a major challenge during drug development. Off-target interactions are often the cause of adverse drug reactions (ADRs) in animal models or clinical studies. That is why careful characterization and identification of drug candidates' secondary pharmacology profiles early in the drug discovery process might help reduce the incidence of ADRs (Bowes et al., 2012). To identify undesirable off-target activities that could impair the development of CPL207280 (as was the case with TAK-875), we performed a screening of 47 most common off-targets (Safety47 Panel, Eurofins).

Safety47 Panel consists of assays designed to study the activity of several GPCR, transporters, ion channels, nuclear receptors, kinases, and nonkinase enzymes. The experiment was performed at $10 \mu \mathrm{M}$, the concentration 125 times higher than $\mathrm{EC}_{50}$ to the intended therapeutic target. The results of each test in the panel (both agonist and antagonist mode) are considered negative if the activation or inhibition of a given pathway does not exceed $70 \%$ of the control.

The obtained results, summarized in Table 2, showed that CPL207280 neither inhibits nor activates any of the studied pathways. Therefore CPL207280 shall be regarded as a safe compound in terms of biomarkers tested in Safety47 Panel.

\section{Pharmacokinetic Study}

The PK profile of CPL207280 was studied in Wistar Han rats (Fig. 5; Table 3) after administration orally and intravenously at a dose of $3 \mathrm{mg} / \mathrm{kg}$. Compound CPL207280 demonstrated high plasma clearance, resulting in plasma half-life of 1.4 hours. This could be advantageous compared with TAK-875, which, when administered at high doses, induced accumulation of total bile acids in circulation in rats and dogs and caused liver injury accompanied by elevation of alanine aminotransferease (ALT) and bilirubin (Wolenski et al., 2017). Additionally, oral administration of CPL207280 exhibited rapid absorption, high $\mathrm{C}_{\max }$, and high plasma exposure with high bioavailability (63\%) (Table 3).

\section{Efficacy of CPL207280 in Healthy Rodents}

The first proof-of-concept study consisted of a GTT performed in young C57BL6/cmdb male mice. To ensure maximal drug exposure, mice were administered intravenously (tail vein) either the respective compound $(10 \mathrm{mg} / \mathrm{kg}$ ) or the vehicle. Glucose was administered immediately after the injection. Next, blood glucose was measured for up to 120 minutes with a standard glucometer. Both compounds reduced glucose levels in a similar, effective fashion and reduced the AUC 2 times compared with the vehicle (Fig. 6). No adverse effects, including changes in the animals' behavior, were observed during the study.

We further tested whether a single dose of CPL207280 can improve a postprandial glucose range after consecutive glucose

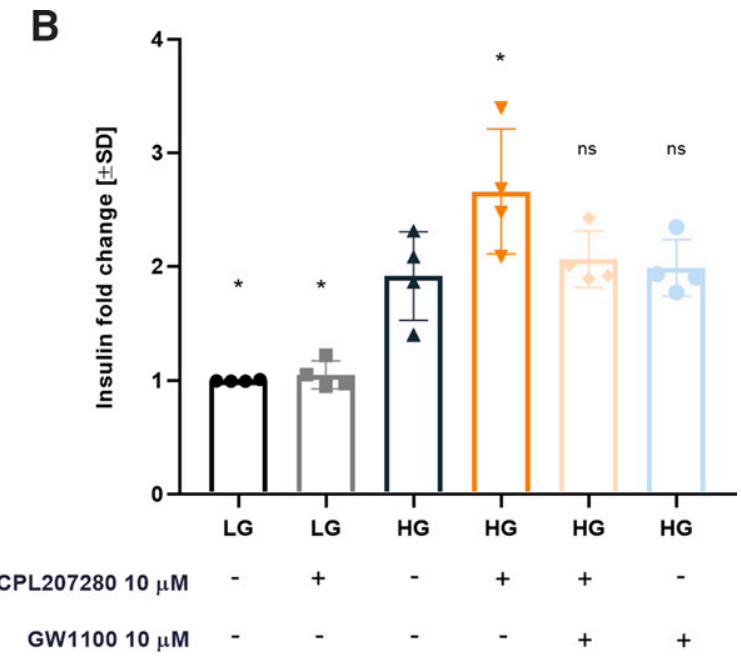

Fig. 3. The effect of CPL207280 on GSIS in vitro. The dose-response relationship of CPL207280 and TAK-875 on insulin secretion in MIN6 cells (A) and effect of GW1100 on CPL207280 potentiation of insulin secretion in INS-1E cells (B) were evaluated after 1-hour incubation; Control (CTR)untreated cells at $20 \mathrm{mM}$ glucose. Data are presented as mean fold changes \pm S.D. of control (untreated cells at $20 \mathrm{mM}$ glucose) from three independent experiments performed in two technical replicates each (A) and four independent experiments performed in two technical replicates each (B). Data were analyzed using one-way ANOVA test (Dunnett's post hoc). ${ }^{*} P<0.05 ;{ }^{*} P<0.01$. LG- low glucose $(2.8 \mathrm{mM}) ; \mathrm{HG}-$ high glucose $(20 \mathrm{mM})$. 

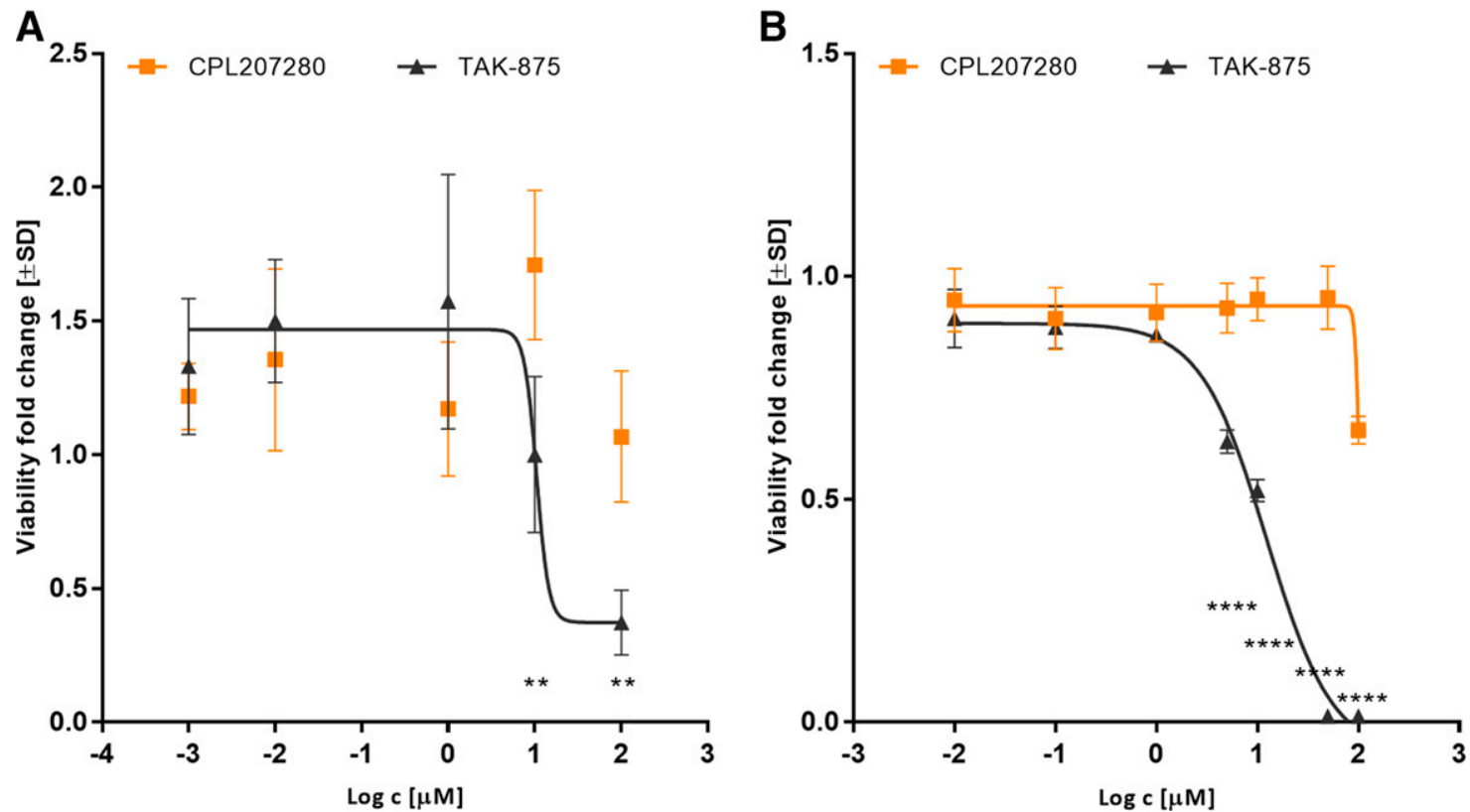

Fig. 4. Cytotoxicity of CPL207280 in insulinoma cells. The viability of MIN6 (A) and INS-1 (B) cells after 48-hour incubation with CPL207280 and TAK-875 at concentrations up to $100 \mu \mathrm{M}$ was evaluated using a luminescence assay. Control cells were treated with $0.5 \%$ DMSO, a solvent. Data are presented as mean fold changes of control cells \pm S.D. from three independent experiments performed in two technical replicates each. Data were analyzed using a two-way ANOVA test (Sidak post hoc). $* * P<0.05 ; * * * P<0.0001$ vs. control.

challenges 6 hours apart. CPL207280 displayed excellent pharmacokinetic properties. The compound after a single $3-\mathrm{mg} / \mathrm{kg}$ dose in rats displayed excellent oral bioavailability $(\% \mathrm{~F}=63)$, a total plasma $\mathrm{C}_{\max }$ of $1.7 \mu \mathrm{g} / \mathrm{ml}$, but also a shorter half-life (1.4 hours versus 4.1 hours for the TAK- 875 compound) (Negoro et al., 2010) (Table 3). To be sure that the concentration of the CPL207280 in the blood allows it to be effective in glucose lowering 6 hours after administration (during the second GTT), a $10-\mathrm{mg} / \mathrm{kg}$ dose was selected for this study. CPL207280 administration in fasted state did not have any effect on glucose levels prior to the glucose tolerance test (30 minutes after CPL207280 administration). No adverse effects, including changes in the animal's behavior, were observed during the study. Interestingly, both CPL207280 and TAK-875 reduced glucose levels significantly after the first, but also after the second, glucose challenge, 6 hours post-drug administration (Fig. 7A). However, only CPL207280 demonstrated a substantial and statistically significant reduction of glucose AUC: by $50 \%$ in the first (Fig. 7D) and 25\% in the second GTT (Fig. 7E). This effect is likely to have resulted from its greater capacity to enhance GSIS, as CPL207280 evoked a substantial elevation of insulin levels in the first hour compared with TAK875 (Fig. 7B). In total, CPL207280 increased the insulin level (presented as AUC) 13.6 times, whereas TAK-875 augmented AUC 5.5 times (Fig. 7F). The increase of plasma insulin levels was most evident 15 and 30 minutes after glucose administration. The therapeutic effect durability suggests that administration of CPL207280 once daily may improve postprandial hyperglycemia in subjects with diabetes throughout the day.

\section{CPL207280 Stimulates Insulin Secretion and Reduces Postprandial Glucose Levels in Diabetic Rats}

Zucker Diabetic Sprague-Dawley Rat Model. To assess the capacity of CPL207280 to improve glycemia in diabetic rodent models and compare it with TAK-875, the reference compound, as well as with glibenclamide, a sulfonylurea available on the market, a dose-response study was performed in a ZDSD rat. A ZDSD rat is a new T2D animal model that was developed to display polygenic obesity and diabetes with an intact leptin pathway (Peterson et al., 2015). Rats aged 12 weeks were fasted for 6 hours, and next the compounds or the vehicle was administered orally at doses $3,10,30$, and $100 \mathrm{mg} / \mathrm{kg}$ b.wt. The $100-\mathrm{mg} / \mathrm{kg}$ dose was also chosen to study the potential risk of hypoglycemia. A glucose bolus was administered 1 hour later. Blood glucose was measured 1 hour and 0.5 hours prior to the glucose bolus administration, as well as during a glucose challenge for up to 4 hours. No adverse effects, including changes in the animals' behavior, were observed during the study. No hypoglycemia events were observed, even for the highest dose of 100 $\mathrm{mg} / \mathrm{kg}$. CPL207280 improved glucose tolerance and reduced glucose AUC already at the lowest dose by $38 \%$, and the effect was dose-independent (no significant differences between doses), as the AUC values for all doses were reduced to a similar degree (Fig. 8, A and B). The improvement in glucose excursions was a result of an increase of GSIS by 207\% (AUC) (Fig. 8D). This effect was also dose-independent (no significant differences between doses were observed), and the second highest observable effect was recorded at the lowest dose. The results suggest that $\mathrm{EC}_{50}$ for CPL207280 in vivo is less than $3 \mathrm{mg} / \mathrm{kg}$. In contrast to healthy animals, the glucose-lowering and insulin augmenting effect was observed before stimulation with a glucose bolus. The increase of plasma insulin levels was most evident at 0.5 hours after the compound administration (before the glucose bolus); however, after the glucose load, it is still at a very high level (significantly different from the vehicle) even 3 hours after the glucose load (for dose 30 and $100 \mathrm{mg} / \mathrm{kg}$ ). 


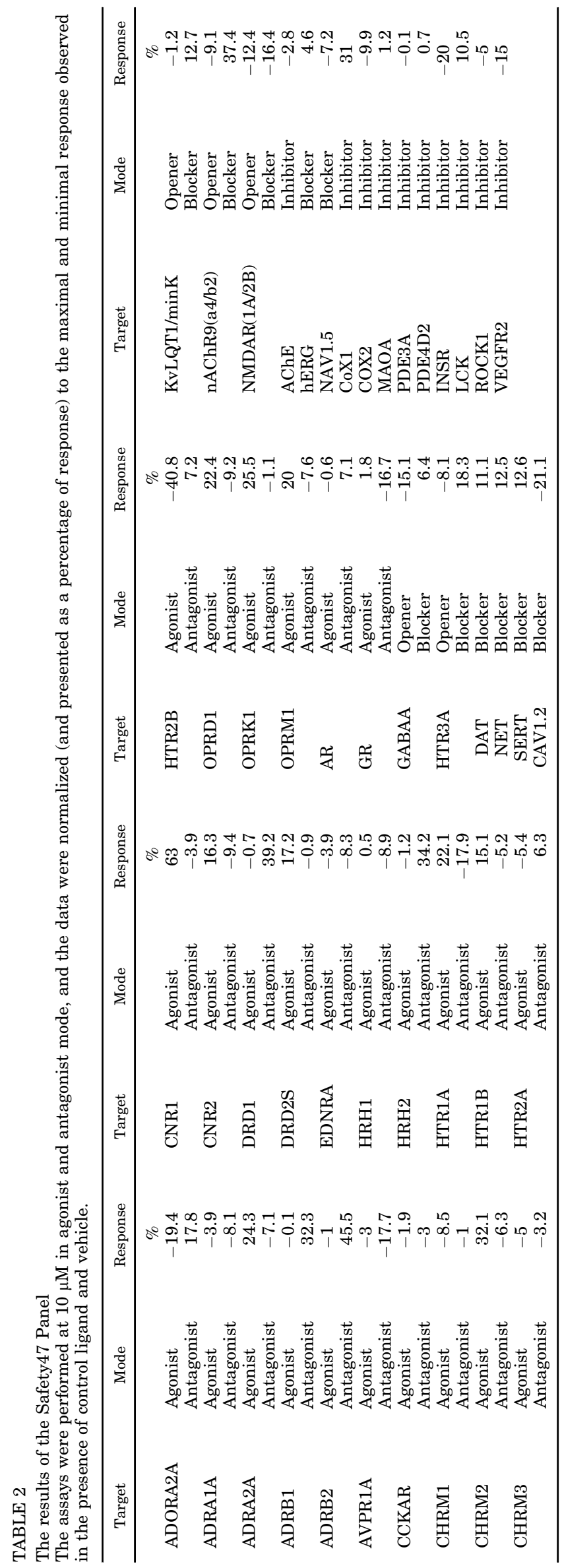

The effect of insulin secretion before the glucose load was expected, as in fasted ZDSD rats, hyperglycemia is still present and permits action of CPL207280 to further propel insulin secretion. Surprisingly, although TAK-875 and glibenclamide increased GSIS by $211 \%$ and $251 \%$, respectively, they failed to improve glucose tolerance or reduce glucose AUC.

Goto-Kakizaki Rat Model. A GK rat is a lean model for T2D that accumulates mutations in the pancreas that cause both $\beta$-cell mass cell death and impairment of their function. In turn, these events cause insufficient insulin secretion and evoke glucose intolerance, leading to diabetes (Al-Awar et al., 2016). Because of the lower potential of insulin secretion expected in this model, although the $3-\mathrm{mg} / \mathrm{kg}$ dose was already effective in the ZDSD rats study, in this study we decided to choose one of the higher doses to get the maximum possible antidiabetic effect.

To study the effectiveness of CPL207280, 14-week-old GK rats were fasted for 6 hours and then were orally administered $30 \mathrm{mg} / \mathrm{kg}$ of the test compounds or the vehicle. At 1 hour later, the animals were challenged with glucose. Blood was sampled from the tail just before drug administration and over the course of the GTT for up to 6 hours. Samples were probed for glucose and insulin measurements. No adverse effects, including changes in the animals' behavior, were observed during the study. Rats treated with CPL207280 showed considerably improved glucose tolerance as compared with vehicle-treated rats (Fig. 9A). The effect emerged at 5 minutes after administration and remained significant for up to 6 hours. CPL207280 lowered the glucose AUC by $30 \%$ (Fig. 9B). Furthermore, rats treated with CPL207280 demonstrated elevated levels of insulin 1 hour after drug administration and after 1 and 2 hours from the glucose challenge (Fig. 9C), but due to impairment in $\beta$-cell function in GK rats, this effect is not as strong (and significant) as in other rat models. In contrast, TAK- 875 given at the same dose demonstrated a moderate effect, which did not reach statistical significance (Fig. 9, A and C). The insulin AUC (counted from the baseline concentration at $t=-1$ hour) increased by $143 \%$ for CPL207280 compared with the control AUC (Fig. 9D). TAK-875 demonstrated a weaker improvement of an insulin AUC and thus lower glucose-normalizing capacity in GK rats.

Zucker Diabetic Fatty Rat Model. A ZDF rat is a popular model for T2D that develops obesity and insulin resistance at a young age. Hyperglycemia, progressively developing with age, is associated with impaired pancreatic $\beta$-cell function, loss of pancreatic $\beta$-cell mass, and decreased responsiveness of liver and extrahepatic tissues to the actions of insulin and glucose (Pal et al., 2016). To study the effectiveness of CPL207280 and TAK-875 in this model, 16 -hour fasting ZDF rats were administered the tested compounds orally at 5 and $15 \mathrm{mg} / \mathrm{kg}$ first and then glucose 30 minutes after a primary dosing. Blood glucose and insulin were determined in each animal up to 2 hours after glucose administration. No adverse effects, including changes in the behavior of the animals, were observed during the study. Serum glucose AUC was decreased significantly in all treatment groups (Fig. 10B) by $81 \%, 77 \%$, and $70 \%$ for CPL207280 at 5 and $15 \mathrm{mg} / \mathrm{kg}$ or TAK-875, respectively. The glucose tolerance in the CPL207280-treated group was even better than that in the normal ZL rats used as a control (Fig. 10, A and B). Serum insulin AUC was enhanced significantly 


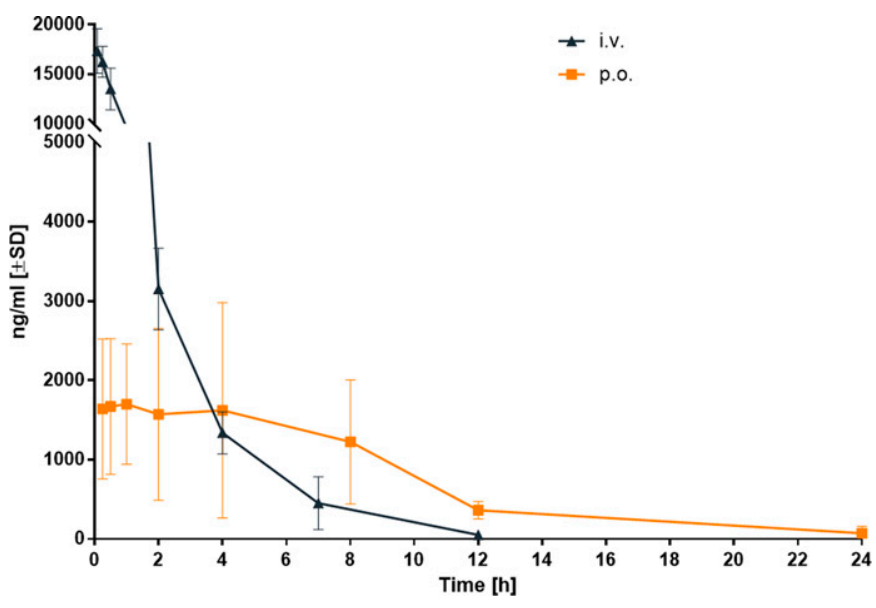

Fig. 5. Mean concentration-time profile of CPL207280. The compound concentration in Wistar Han rats $(n=4 / 5)$ plasma was measured after oral and intravenous administration at $3 \mathrm{mg} / \mathrm{kg}$. Data are presented as means \pm S.D.

and was dose-dependent in the CPL207280-treated group (Fig. 10, C and D). The amount of insulin secreted after CPL207280 administration was elevated significantly 15 minutes after glucose load; therefore, despite the maximum insulin concentration observed 30 minutes after glucose load (Fig. 10C), the blood glucose level decreased already after 15 minutes (Fig. 10A).

\section{Discussion}

GPR40 (or FFA1) belongs to the GPCRs family and is highly expressed in $\beta$-pancreas cells. Activation of GPR40 by medium- and long-chain FFAs or synthetic compounds has been reported to enhance insulin secretion in a glucosedependent manner and is considered to have potential as a novel target for the treatment of T2D (Mancini and Poitout, 2015). The present results showed that CPL207280 increased $\mathrm{Ca}^{2+}$ in CHO cells expressing human GPR40 with a higher potency than TAK-875 (the reference compound; Fig. 2) and had no impact on cells expressing related receptors: GPR41 (FFA2), GPR43 (FFA3), GPR55, GPR119, GPR120, GPR142 (Table 1). Despite activation of GPR40/FFA1 by some thiazolidinediones acting as PPAR $\gamma$ agonists (Kotarsky et al., 2003), CPL207280 was found to not affect PPAR $\alpha$ or PPAR $\gamma$ in a nuclear receptor reporter activation assay (Table 1). Collectively, these data demonstrate that CPL207280 is a potent and selective GPR40/FFA1 agonist with a mechanism distinct from those of other clinically available oral insulinotropic drugs, including SUs and dipeptidyl peptidase-4 inhibitors (Winzell and Ahrén, 2007).

Long-chain FFAs, natural ligands for GPR40, acutely enhance insulin secretion, and chronic exposure to FFAs causes toxicity, such as death or dysfunction of $\beta$-cells (Sharma

TABLE 3

Pharmacokinetic parameters for CPL207280 in fasted Wistar Han rats

\begin{tabular}{lccccc}
\hline & $\mathrm{C}_{\max }$ & $\mathrm{T}_{\max }$ & $\mathrm{AUC}$ & $t_{1 / 2}$ & $\mathrm{~F}$ \\
\hline & $n g / m l$ & $\min$ & $n g h / m l$ & $h$ & $\%$ \\
Oral & 1699 & 60 & 17,559 & 0.49 & 63 \\
Intravenous & 17,365 & 5 & 27,128 & & \\
\hline
\end{tabular}

$t_{1 / 2}$, half-life. and Alonso, 2014). Therefore, GPR40/FFA1 has been considered as a potential mediator of lipotoxicity (Steneberg et al., 2005), but a number of experimental observations do not support the central role of GPR40/FFA1 in lipotoxicity (Latour et al., 2007; Kebede et al., 2008; Lan et al., 2008; Alquier and Poitout, 2009). In the present study, we did not observe any signs of toxicity in insulinoma cells treated for 48 hours, in contrast to TAK-875, which reduced viability of rat and mouse insulinoma cells at higher concentrations, which suggests a narrower window of safety (Fig. 4). These data are in contrast to the previously presented results (Tsujihata et al., 2011) that showed no evidence of $\beta$-cell dysfunction in vitro after the prolonged exposure to TAK-875. Authors showed that TAK-875 did not affect caspase 3/7 activity after prolonged 72-hour incubation. Nonetheless, it must be emphasized that although caspase 3/7 activity is widely accepted as a reliable indicator for cell apoptosis, it may not reflect other types of death potentially mediated by TAK-875, e.g., necrosis. Additionally, 1\% BSA in culture medium was used in the TAK-875 study. In this paper, we presented data from experiments in which FBS/BSA-free media were used to determine the cytotoxicity in each cell line. It is important to note that TAK- 875 is highly bound to plasma proteins (99.8\% in rats) (Otieno et al., 2018), so the addition of BSA to the caspase $3 / 7$ activity experiment could cause the TAK-875 binding to BSA, and therefore the cytotoxicity effect could be attenuated. The mechanism underlining TAK-875driven cell death has yet to be elucidated.

One of the main challenges in decreasing the high attrition rate in the drug discovery and development process is achieving a good balance between drug efficacy and potential adverse effects as early as possible (Bowes et al., 2012). The example of TAK-875, the development of which was terminated during phase III of clinical trials due to hepatotoxicity, showed that the safety profile should also be carefully studied as early as at the structure optimization process. Therefore, early profiling against a plethora of targets that are known to underlie ADRs, such as hERG (which is linked to cardiac arrhythmias) or the 5-hydroxytryptamine (serotonin) receptor $2 \mathrm{~B}$ (which is linked to cardiac valvulopathy) was performed in this study and confirmed CPL207280's selectivity of and lack of interaction with the tested off-targets (Table 2).

One of the proposed mechanisms of TAK- 875 hepatotoxicity after multiple administrations in human is its long halflife and accumulation in the liver at high doses (Wolenski et al., 2017). Additionally, this half-life is longer in patients with type 2 diabetes compared with healthy volunteers (51.6 hours vs. 36.6 hours for a 50-mg dose) (Leifke et al., 2012; Naik et al., 2012). Results obtained from a pharmacokinetic study on Wistar Han rats demonstrated that in addition to the high bioavailability and concentration in the blood (Table 3), CPL207280 is characterized by a shorter half-life. This reduced the risk of accumulation during long-term administration. Despite this shorter half-life for CPL207280, its high activity still provides an antidiabetic effect, which has been demonstrated in animal studies.

Because CPL207280 exhibited a favorable effect as an insulin secretagogue in mouse insulinoma cells, subsequently, the glucose-lowering effects of CPL207280 were evaluated in vivo during GTT in healthy rodents. A single administration of CPL207280 reduced plasma glucose levels during GTT in healthy rats after both the first and the second glucose challenge (Fig. 7, A, C, D, and E). Notably, the administration of 

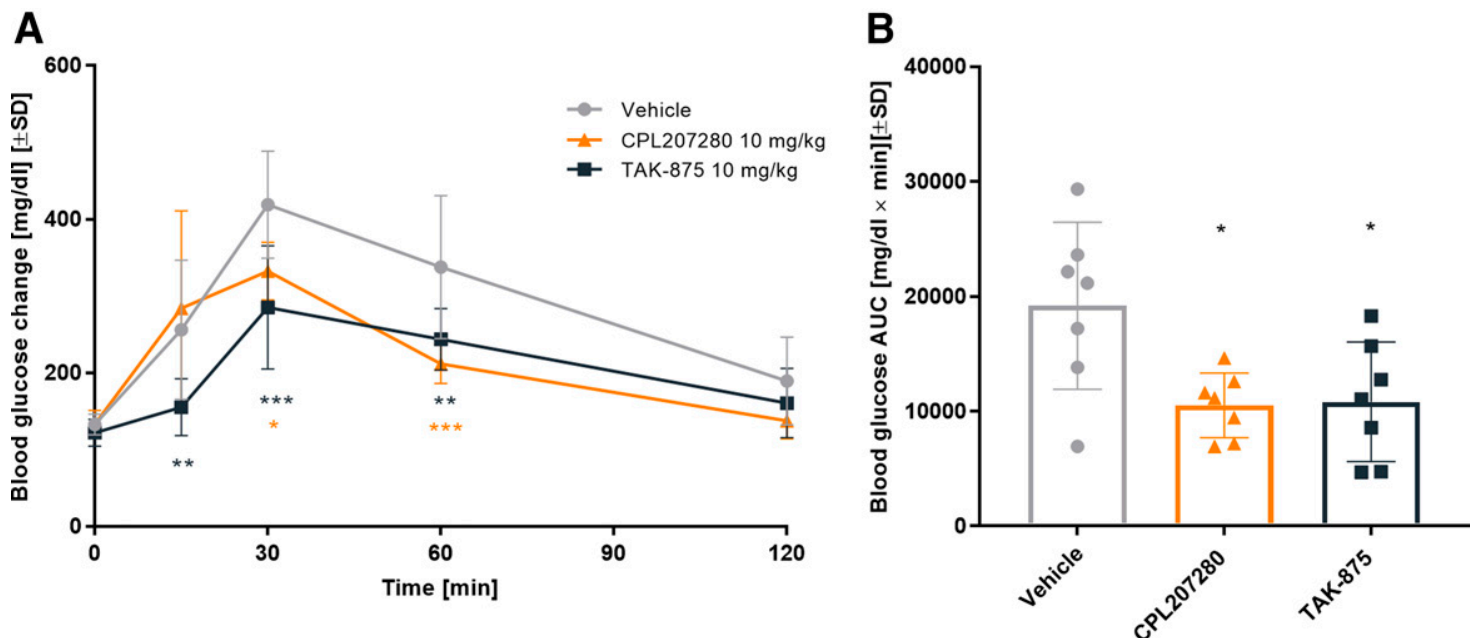

Fig. 6. Improvement of glucose tolerance in C57BL6/cmdb mice treated with CPL207280. The effect of intravenous administration of CPL207280 or TAK-875 on blood glucose concentration after a glucose challenge in C57BL6/cmdb mice (A) and corresponding AUCs (B) was evaluated during GTT. Data are presented as means \pm S.D. $(n=7)$. The results were analyzed using one-way ANOVA (B) or two-way ANOVA (A) with Dunnett's post hoc test vs. control; * $P<0.05, * * P<0.01, * * * P<0.001$.

CPL207280 in the fasted state did not have any effect on glucose levels prior to the GTT (Fig. 7A) in contrast to diabetic models, in which we observed stimulation of insulin secretion and glucose lowering (Figs. 8, A and C, 9, A and C, and 10, A and $\mathrm{C}$ ) before the glucose load. Collectively, these observations indicate glucose dependence of CPL207280 and TAK-875 action. This corresponds with the results of the previous study demonstrating that TAK- 875 did not increase the risk of hypoglycemia in patients with T2D (Leifke et al., 2012).
As postprandial hyperglycemia caused by insufficient insulin secretion in response to blood glucose levels is observed in patients with T2D (Wajchenberg, 2007; Leahy, 2005), we tested CPL207280 in GTT in different diabetic rat models (GK, ZDSD, ZDF). Provided that the T2D rat models used in this study represent different stages of diabetes or different phenotypes typically observed in humans, this study allows us to compare CPL207280 with other T2D drugs available on the market and meet the expectations of patients with T2D.
A

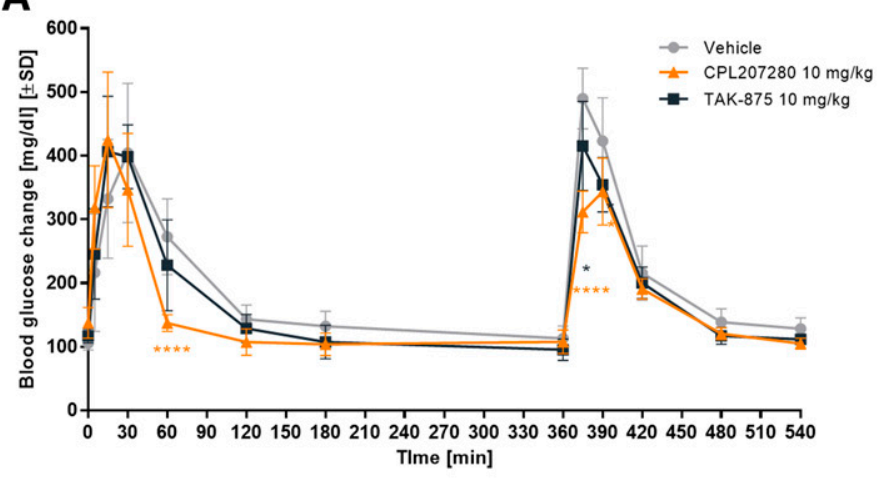

B

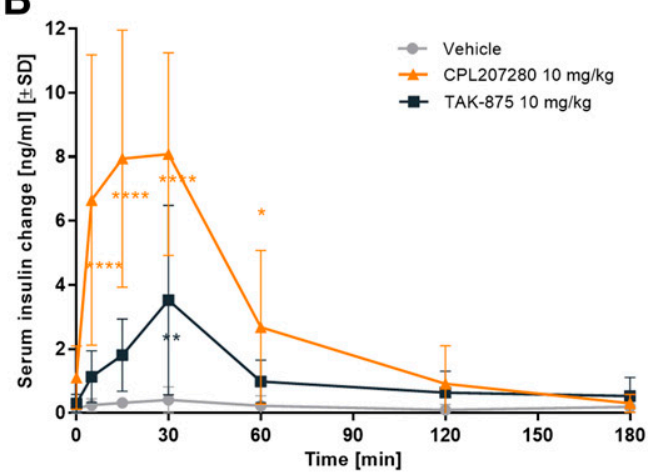

C

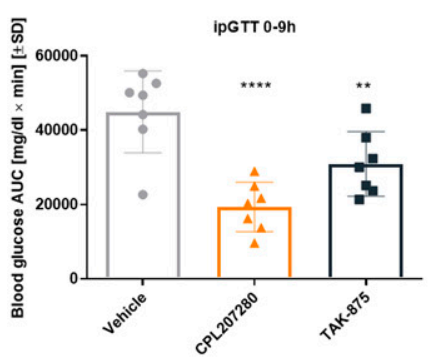

D

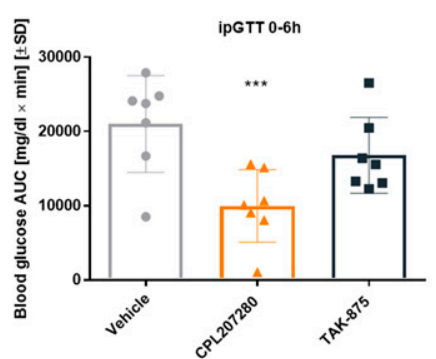

E

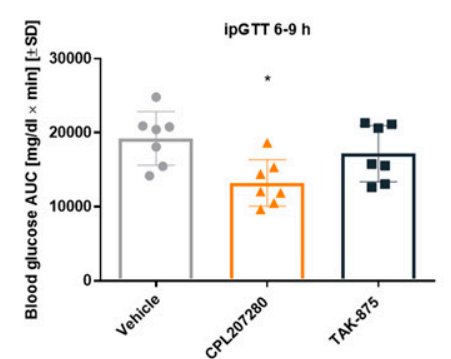

$\mathbf{F}$

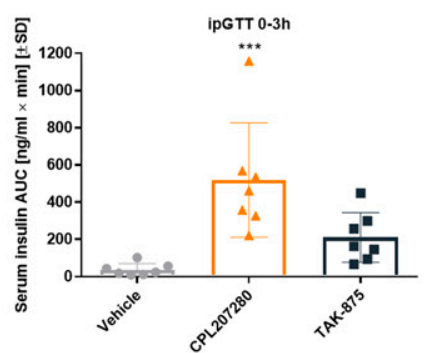

Fig. 7. Improvement of glucose tolerance and GSIS in two consecutive GTTs in Wistar Han rats treated with CPL207280. The effect of oral administration of $10 \mathrm{mg} / \mathrm{kg}$ CPL207280 or TAK-875 on blood glucose and insulin concentration after two consecutive glucose challenges in fasted Wistar Han rats was evaluated during GTT. The graphs show glucose concentration (A); insulin concentration (B); glucose AUCs in both (C), first (D), and second (E) GTTs; and insulin AUC in first GTT (F). Data are presented as mean concentrations \pm S.D. $(n=7)$. The results were analyzed using one-way ANOVA (C-F) or two-way ANOVA (A and B) with Dunnett's post hoc test vs. control; * $P<0.05, * * P<0.01, * * * P<0.001,{ }^{* * * * P}<0.0001$. 

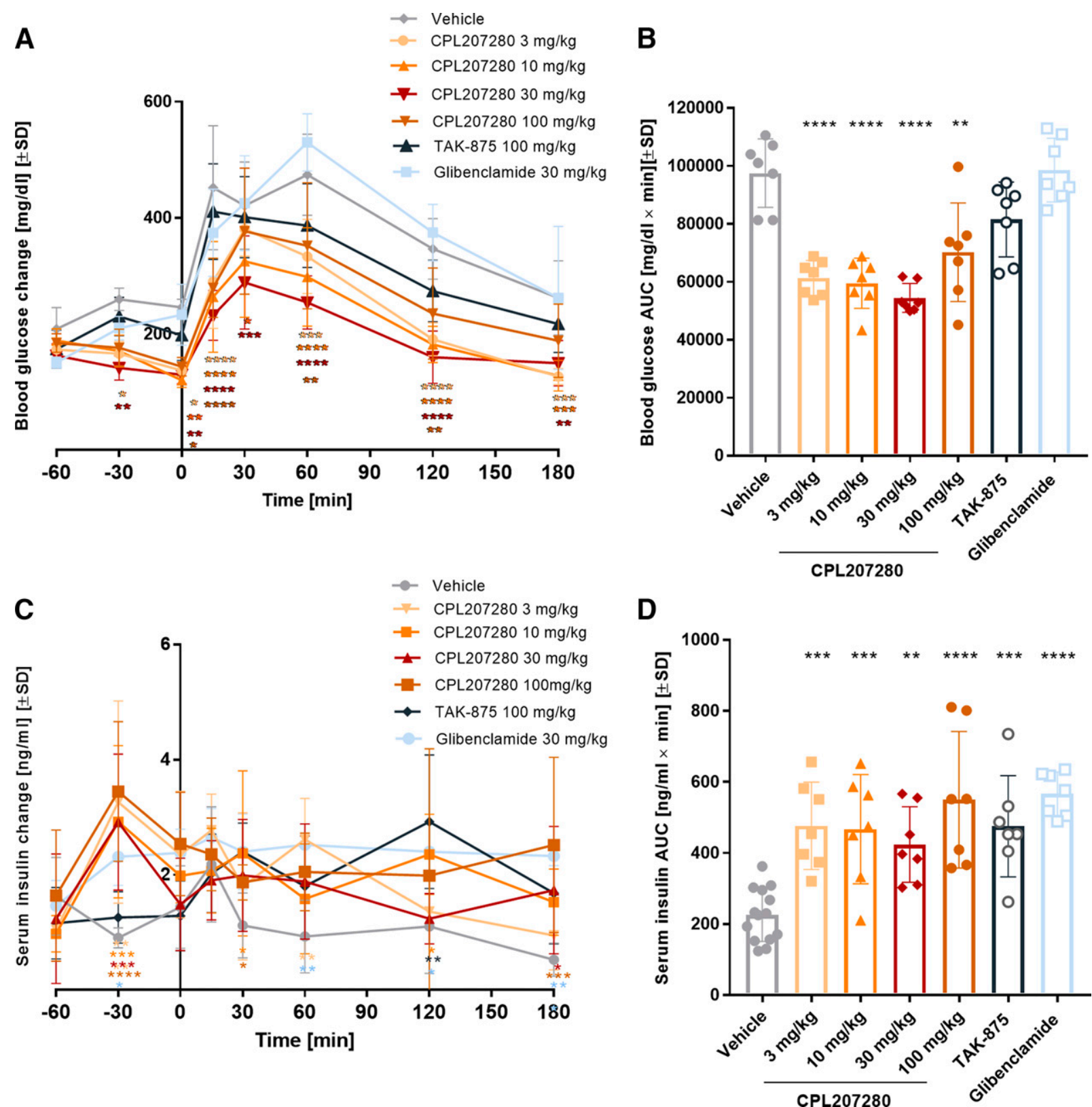

Fig. 8. Improvement of glucose tolerance and GSIS in diabetic ZDSD rats treated with CPL207280. The effect of oral administration of CPL207280 or TAK-875 on blood glucose and insulin concentration after a glucose challenge in fasted ZDSD rats was evaluated during GTT. Graphs present glucose concentrations (A) and glucose AUC (B), insulin concentrations (C), and corresponding insulin AUC (D). Data are presented as means \pm S.D. $(n=7)$. The results were analyzed using one-way ANOVA (B and D) or two-way ANOVA (A and C) with Dunnett's post hoc test vs. control. $* P<0.05 ; * * P<0.01 ; * * * P<0.001 ; * * * P<0.0001$

A ZDSD rat model was developed as an alternative to ZDF rats, which are a gold standard for diabetes studies. However, despite sharing many of the characteristics of the human condition, ZDF rats have a genetic defect in the leptin receptor, which is considered a disadvantage, and that makes them an artificial model (Peterson et al., 2015). Unlike ZDF rats, a ZDSD model exhibits type 2 diabetes with a long prediabetic condition in the presence of an intact leptin pathway. Animals demonstrate obesity, impaired glucose tolerance, insulin resistance, dyslipidemia, and prediabetic hypertension-symptoms reflecting the initial stage of diabetes development very well (Weir and Bonner-weir, 2004). In ZDSD rats, CPL207280 enhanced insulin secretion and reduced plasma glucose levels in GTT but also reduced blood glucose prior to the GTT in all tested concentrations (Fig. 8). Interestingly, glibenclamide used as a control, representing sulfonylureas, a class of medicines commonly used in T2D therapy, also significantly increased insulin concentration, immediately after administration, but was not effective in lowering glucose concentrations (Fig. 8B). Although a ZDF rat as a T2D model has some disadvantages, it also has many advantages and is one of the most popular models employed to demonstrate the antidiabetic activity of new compounds. In a ZDF rat, which develops obesity and high insulin resistance at a young age and then, with aging, progressively develops hyperglycemia, CPL207280 also 
A
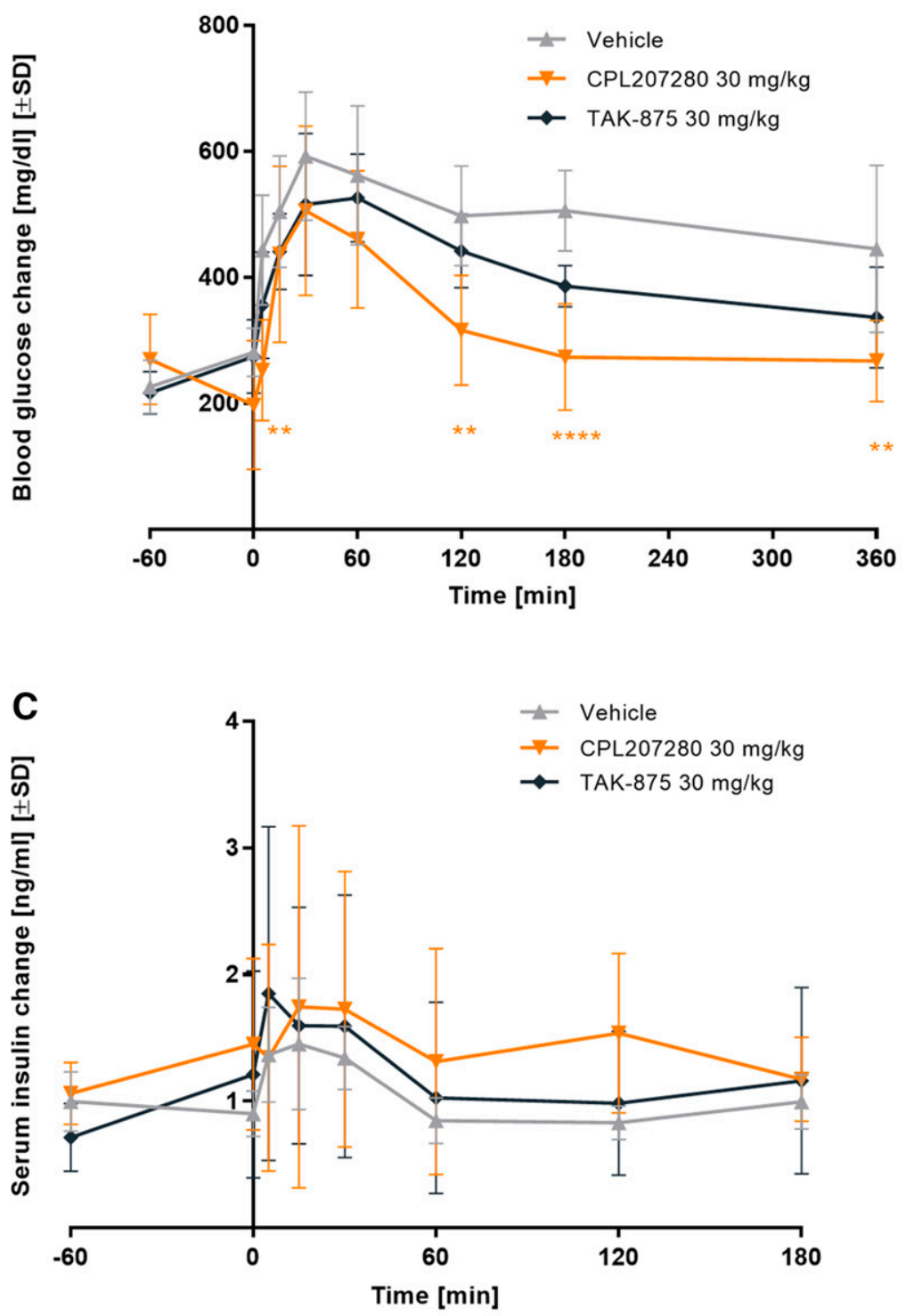

B
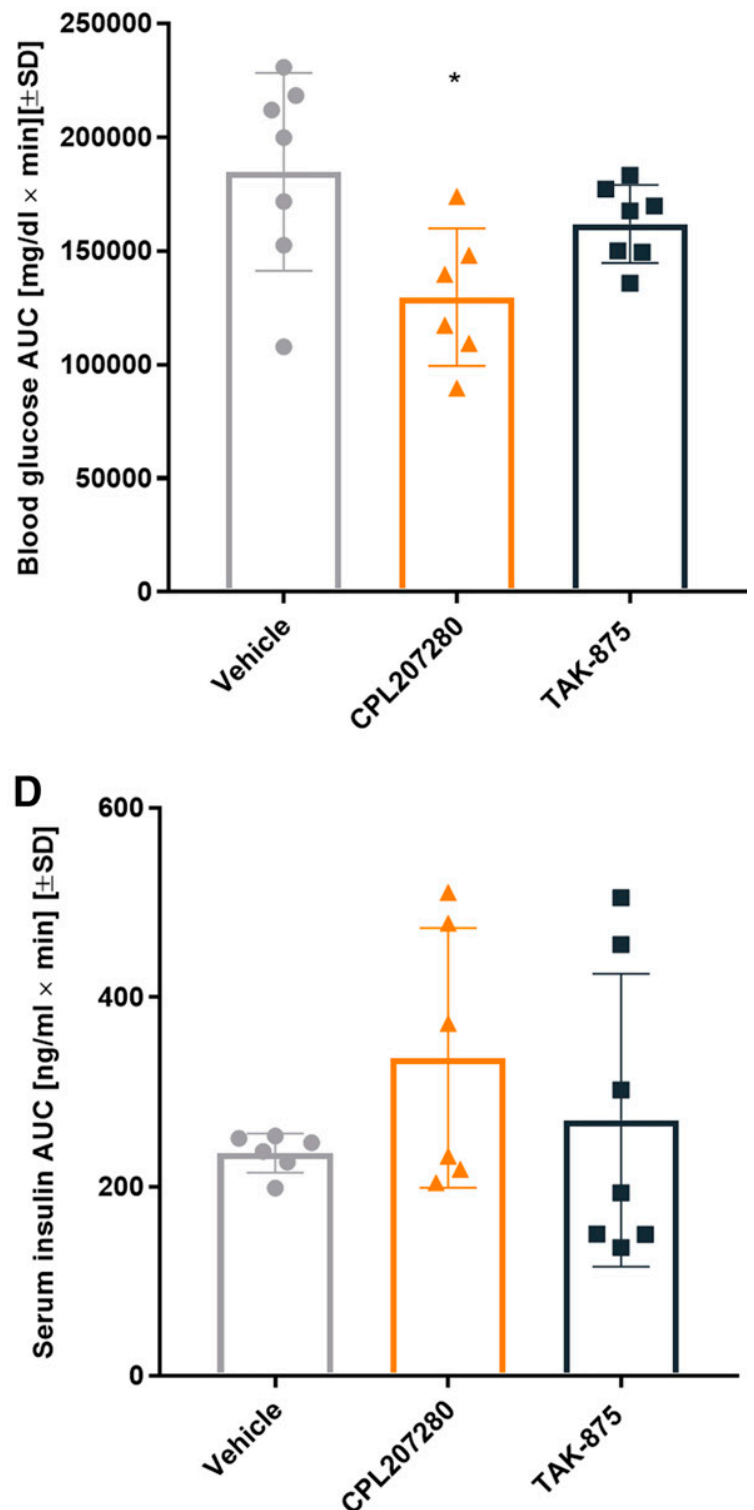

Fig. 9. Improvement of glucose tolerance and GSIS in diabetic GK rats treated with CPL207280. The effect of oral administration of CPL207280 or TAK- 875 on blood glucose and insulin concentration after a glucose challenge in fasted GK rats was evaluated during GTT. The graphs show glucose concentrations (A) and glucose AUC (B), insulin concentrations (C), and corresponding insulin AUC (D). Data are presented as means \pm S.D. $(n=7)$ and were analyzed using one-way ANOVA (B and D) or by use of two-way ANOVA (A and C) with Dunnett's post hoc test vs. control. $* P<0.05 ; * * P<0.01 ; * * * P<0.001 ; * * * * P<0.0001$.

improved glucose tolerance of the subjects and induced their insulin secretion in a dose-dependent manner. Our findings support the previous results (Ito et al., 2013) showing that administration of TAK-875 in ZDF rats resulted in an improvement of glucose tolerance and fasting hyperglycemia (Fig. 10).

During progression toward diabetes, when the $\beta$-cell mass becomes inadequate at some critical point, glucose levels increase over a relatively short time (Weir and Bonner-weir, 2004). Some similarities to this diabetes stage are represented by the GK rat, which is a nonobese T2D model with a defective $\beta$-cell number and function, leading to insufficient insulin secretion. As GPR40 agonists act as insulin secretagogues, we explored whether CPL207280 can regulate glucose levels also in this diabetic phenotype. Interestingly, in this model, we also observed that a single oral administration of CPL207280 enhanced insulin secretion and reduced plasma glucose levels during GTT but also has a glucose-lowering effect prior to the glucose tolerance test (Fig. 9). This finding confirmed that a glucose-lowering effect exerted through a GPR40 agonist occurs only under the condition of an elevated glucose level in the blood, unlike SUs that act on $\beta$-cells to continuously secrete insulin independently of blood glucose levels, resulting in hypoglycemia. It is worth noting that, among the tested diabetic models, GK rats showed the least response to CPL207280 in terms of insulin AUC (Fig. 9). This observation suggests that treatment with CPL207280 may be less effective in those individuals whose $\beta$-cell function is substantially impaired. 
A

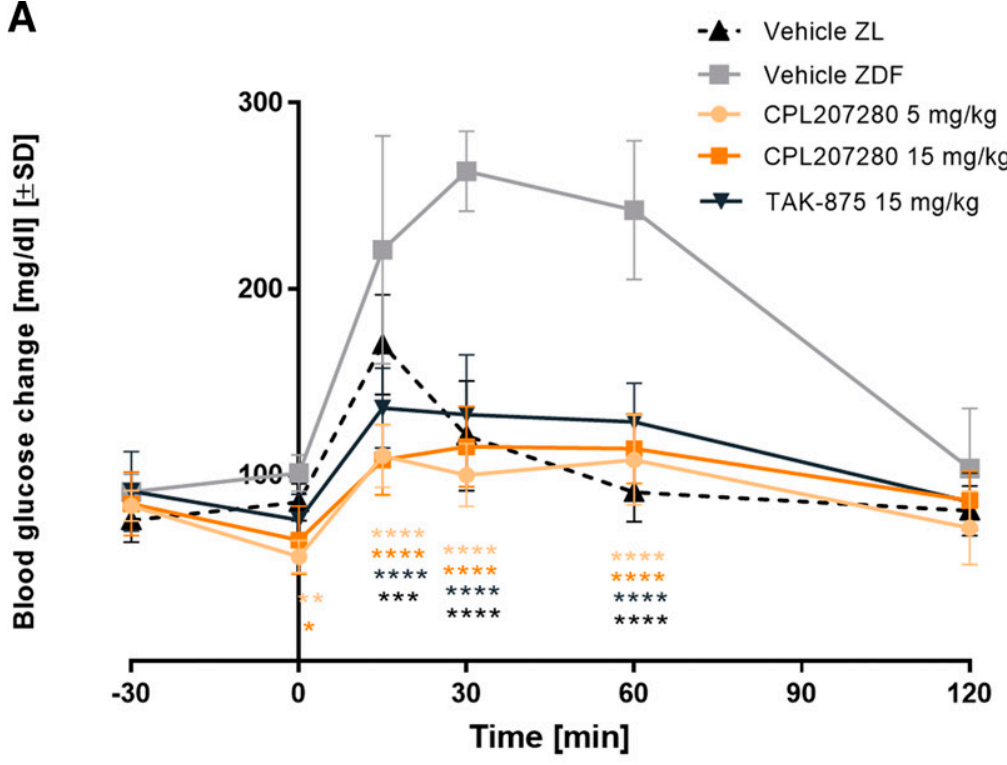

B

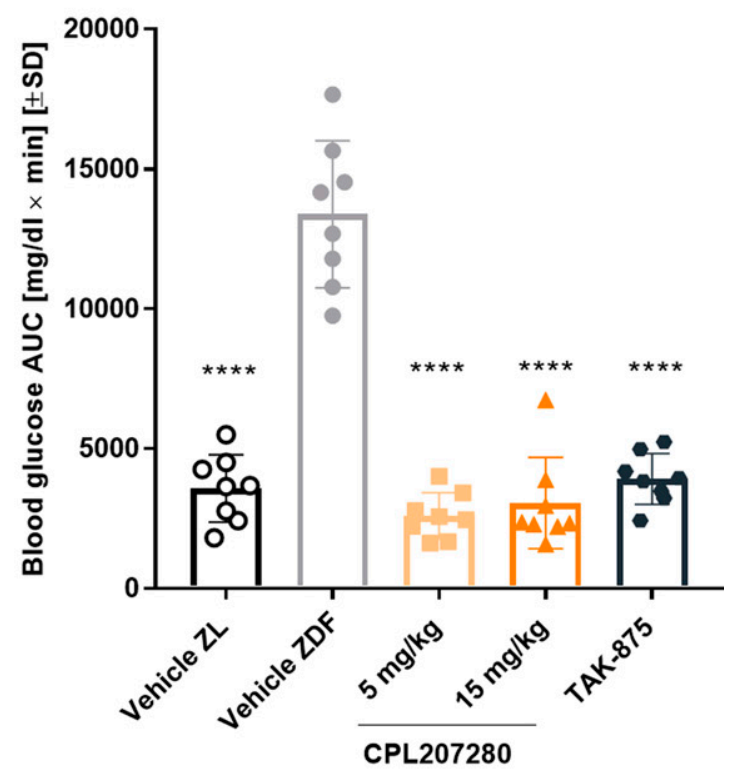

D

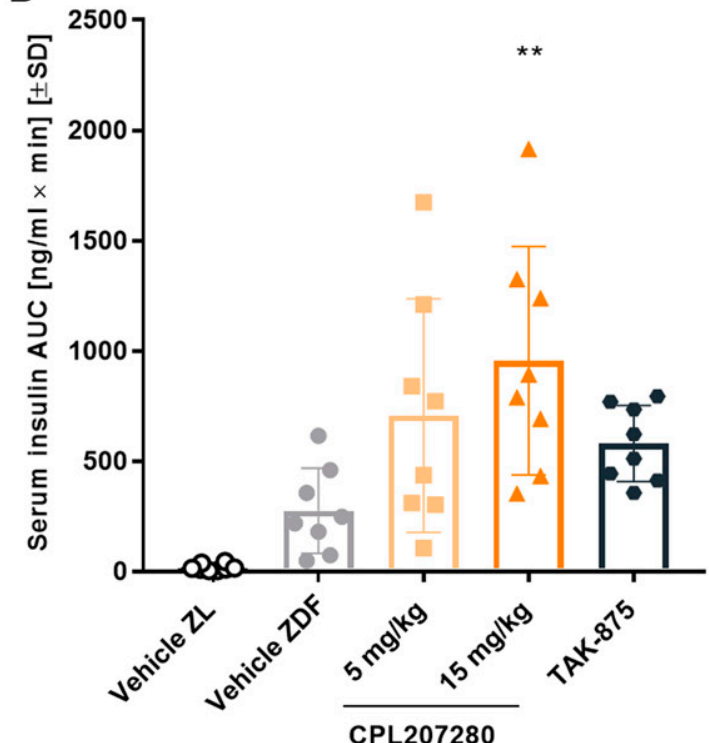

Fig. 10. Improvement in glucose tolerance and GSIS in diabetic ZDF rats treated with CPL207280. The effect of oral administration of CPL207280 or TAK-875 on blood glucose and insulin concentration after a glucose challenge in fasted ZDF rats was evaluated during GTT. The graphs show glucose concentrations (A) and glucose AUC (B), insulin concentrations (C), and corresponding AUC (D). Data are presented as means \pm S.D. $(n=8)$. The results were analyzed using one-way ANOVA with (B and D) or two-way ANOVA (A and C) with Dunnett's post hoc test vs. control. $* P<0.05 ; * * P<0.01 ; * * * P<0.001 ; * * * P<0.0001$.

Glucose-stimulated insulin secretion in vivo typically follows a biphasic time course and consists of a rapid and transient first phase followed by a sustained second phase (Barbosa et al., 1998; Rorsman et al., 2000). The first phase of insulin secretion is mediated by the pool of insulin secretory granules located near the plasma membrane, whereas the second phase involves the transport of insulin granules from an intracellular reserve pool to the plasma membrane (Wang and Thurmond, 2009). It was examined that GPR40 agonists potentiated the second phase of insulin secretion preferentially in pancreatic islets (Ferdaoussi et al., 2012).
The high and sustained insulin level observed in diabetic animal studies (Figs. 8C, 9C, and 10C) is probably the second phase of insulin release. The first phase of insulin secretion in response to the elevated blood glucose level in diabetic animal models occurred just after compound administration but was unnoticed. Therefore, we observed the glucose-lowering effect even before the insulin peak (Figs. 9A and 10A) or even before the glucose bolus administration (Fig. 8A).

In conclusion, in the present study, we demonstrate that CPL207280 exhibited favorable pharmacological effects in various T2D animal models representing and relevant to 
different diabetes phenotypes without a detectable risk of hypoglycemia. Additionally, CPL207280 successfully completed a phase I trial and met its primary endpoint with no adverse safety signals detected (NCT04622111). Therefore, we propose the CPL207280 compound as a novel, glucoselowering agent that overcomes the unmet medical needs of patients with T2D.

\section{Authorship Contributions}

Participated in research design: Bazydlo-Guzenda, Buda, HuczKalitowska, Teska-Kaminska, Pieczykolan.

Conducted experiments: Bazydlo-Guzenda, Buda, Vialichka.

Performed data analysis: Bazydlo-Guzenda, Buda, Matloka.

Contributed new reagents or analytic tools: Mach, Stelmach, Dzida, Smuga, Dubiel.

Wrote or contributed to the writing of the manuscript: BazydloGuzenda, Buda, Kaminska, Wieczorek, Pieczykolan.

\section{References}

Al-Awar A, Kupai K, Veszelka M, Szűcs G, Attieh Z, Murlasits Z, Török S, Pósa A, and Varga C (2016) Experimental diabetes mellitus in different animal models. $J$ Diabetes Res 2016:9051426.

Alquier T and Poitout V (2009) GPR40: good cop, bad cop? Diabetes 58:1035-1036.

Barbosa RM, Silva AM, Tomé AR, Stamford JA, Santos RM, and Rosário LM (1998) Control of pulsatile 5-HT/insulin secretion from single mouse pancreatic islets by intracellular calcium dynamics. J Physiol 510:135-143.

Bowes J, Brown AJ, Hamon J, Jarolimek W, Sridhar A, Waldron G, and Whitebread S (2012) Reducing safety-related drug attrition: the use of in vitro pharmacological profiling. Nat Rev Drug Discov 11:909-922 Nature Publishing Group.

Briscoe CP, Tadayyon M, Andrews JL, Benson WG, Chambers JK, Eilert MM, Ellis C, Elshourbagy NA, Goetz AS, Minnick DT, et al. (2003) The orphan G protein-coupled receptor GPR40 is activated by medium and long chain fatty acids. $J$ Biol Chem 278:11303-11311.

Burant CF, Viswanathan P, Marcinak J, Cao C, Vakilynejad M, Xie B, and Leifke E (2012) TAK-875 versus placebo or glimepiride in type 2 diabetes mellitus: a phase 2, randomised, double-blind, placebo-controlled trial. Lancet 379:1403-1411.

Ferdaoussi M, Bergeron V, Zarrouki B, Kolic J, Cantley J, Fielitz J, Olson EN, Prentki M, Biden T, MacDonald PE, et al. (2012) G protein-coupled receptor (GPR)40-dependent potentiation of insulin secretion in mouse islets is mediated by protein kinase D1. Diabetologia 55:2682-2692.

Fujiwara K, Maekawa F, and Yada T (2005) Oleic acid interacts with GPR40 to induce $\mathrm{Ca} 2+$ signaling in rat islet $\beta$-cells: mediation by PLC and L-type $\mathrm{Ca} 2+$ channel and link to insulin release. Am J Physiol Endocrinol Metab 289:E670-E677.

Hara T, Hirasawa A, Ichimura A, Kimura I, and Tsujimoto G (2011) Free fatty acid receptors FFAR1 and GPR120 as novel therapeutic targets for metabolic disorders. J Pharm Sci 100:3594-3601.

Ito R, Tsujihata Y, Matsuda-Nagasumi K, Mori I, Negoro N, and Takeuchi K (2013) TAK-875, a GPR40/FFAR1 agonist, in combination with metformin prevents progression of diabetes and $\beta$-cell dysfunction in Zucker diabetic fatty rats. $\mathrm{Br} J$ Pharmacol 170:568-580.

Ito R, Tsujihata Y, Suzuki M, Miyawaki K, Matsuda K, and Takeuchi K (2016) Fasiglifam/TAK-875, a selective GPR40 agonist, improves hyperglycemia in rats unresponsive to sulfonylureas and acts additively with sulfonylureas. J Pharmacol Exp Ther 357:217-227.

Itoh Y and Hinuma S (2005) GPR40, a free fatty acid receptor on pancreatic $\beta$ cells, regulates insulin secretion. Hepatol Res 33:171-173.

Itoh Y, Kawamata Y, Harada M, Kobayashi M, Fujii R, Fukusumi S, Ogi K, Hosoya M, Tanaka Y, Uejima H, et al. (2003) Free fatty acids regulate insulin secretion from pancreatic $\beta$ cells through GPR40. Nature 422:173-176.

Iwakura T, Fujimoto S, Kagimoto S, Inada A, Kubota A, Someya Y, Ihara Y, Yamada Y, and Seino Y (2000) Sustained enhancement of $\mathrm{Ca}(2+)$ influx by glibenclamide induces apoptosis in RINm5F cells. Biochem Biophys Res Commun 271:422-428.

Kebede M, Alquier T, Latour MG, Semache M, Tremblay C, and Poitout V (2008) The fatty acid receptor GPR40 plays a role in insulin secretion in vivo after high-fat feeding. Diabetes 57:2432-2437.

Kotarsky K, Nilsson NE, Olde B, and Owman C (2003) Progress in methodology. Improved reporter gene assays used to identify ligands acting on orphan seventransmembrane receptors. Pharmacol Toxicol 93:249-258.

Lan H, Hoos LM, Liu L, Tetzloff G, Hu W, Abbondanzo SJ, Vassileva G, Gustafson EL, Hedrick JA, and Davis HR (2008) Lack of FFAR1/GPR40 does not protect mice from high-fat diet-induced metabolic disease. Diabetes 57:2999-3006.
Latour MG, Alquier T, Oseid E, Tremblay C, Jetton TL, Luo J, Lin DCH, and Poitout V (2007) GPR40 is necessary but not sufficient for fatty acid stimulation of insulin secretion in vivo. Diabetes 56:1087-1094.

Leahy JL (2005) Pathogenesis of type 2 diabetes mellitus. Arch Med Res 36:197-209. Leifke E, Naik H, Wu J, Viswanathan P, Demanno D, Kipnes M, and Vakilynejad M 2012) A multiple-ascending-dose study to evaluate safety, pharmacokinetics, and pharmacodynamics of a novel GPR40 agonist, TAK-875, in subjects with type 2 diabetes. Clin Pharmacol Ther 92:29-39.

Li X, Zhong K, Guo Z, Zhong D, and Chen X (2015) Fasiglifam (TAK-875) inhibits hepatobiliary transporters: a possible factor contributing to fasiglifam-induced liver injury. Drug Metab Dispos 43:1751-1759.

Mach M, Dzida R, Smuga D, Stelmach F, Matloka M, Bazydlo K, Dubiel K, Wieczorek M, and Pieczykolan JS. 3-phenyl-4-hexynoic acid derivatives as GPR40 agonists, PCT Patent Application, WO 2019/134984 A1.

Maedler K, Carr RD, Bosco D, Zuellig RA, Berney T, and Donath MY (2005) Sulfonylurea induced $\beta$-cell apoptosis in cultured human islets. J Clin Endocrinol Metab 90:501-506.

Mancini AD and Poitout V (2015) GPR40 agonists for the treatment of type 2 diabetes: life after 'TAKing' a hit. Diabetes Obes Metab 17:622-629.

Marcinak J, Cao C, Lee D, and Ye Z (2017) Fasiglifam for glycaemic control in people with type 2 diabetes: A phase III, placebo-controlled study. Diabetes Obes Metab 19:1714-1721.

Nagasumi K, Esaki R, Iwachidow K, Yasuhara Y, Ogi K, Tanaka H, Nakata M, Yano T, Shimakawa K, Taketomi S, et al. (2009) Overexpression of GPR40 in pancreatic $\beta$-cells augments glucose-stimulated insulin secretion and improves glucose tolerance in normal and diabetic mice. Diabetes 58:1067-1076.

Naik H, Vakilynejad M, Wu J, Viswanathan P, Dote N, Higuchi T, and Leifke E (2012) Safety, tolerability, pharmacokinetics, and pharmacodynamic properties of the GPR40 agonist TAK-875: results from a double-blind, placebo-controlled single oral dose rising study in healthy volunteers. $J$ Clin Pharmacol 52:1007-1016.

Negoro N, Sasaki S, Mikami S, Ito M, Suzuki M, Tsujihata Y, Ito R, Harada A, Takeuchi K, Suzuki N, et al. (2010) Discovery of TAK-875: a potent, selective, and orally bioavailable GPR40 agonist. ACS Med Chem Lett 1:290-294.

Otieno MA, Snoeys J, Lam W, Ghosh A, Player MR, Pocai A, Salter R, Simic D, Skaggs H, Singh B, et al. (2018) Fasiglifam (TAK-875): mechanistic investigation and retrospective identification of hazards for drug induced liver injury. Toxicol Sci 163:374-384.

Pal A, Rhoads DB, and Tavakkoli A (2016) Effect of portal glucose sensing on systemic glucose levels in SD and ZDF Rats. PLoS One 11:e0165592.

Peterson RG, Jackson CV, Zimmerman K, de Winter W, Huebert N, and Hansen MK (2015) Characterization of the ZDSD rat: a translational model for the study of metabolic syndrome and type 2 diabetes. J Diabetes Res 2015:487816.

Poitout V, Amyot J, Semache M, Zarrouki B, Hagman D, and Fontés G (2010) Glucolipotoxicity of the pancreatic beta cell. Biochim Biophys Acta 1801:289-298.

Rorsman P, Eliasson L, Renström E, Gromada J, Barg S, and Göpel S (2000) The cell physiology of biphasic insulin secretion. News Physiol Sci 15:72-77.

Schnell S, Schaefer M, and Schöfl C (2007) Free fatty acids increase cytosolic free calcium and stimulate insulin secretion from $\beta$-cells through activation of GPR40. Mol Cell Endocrinol 263:173-180.

Sharma RB and Alonso LC (2014) Lipotoxicity in the pancreatic beta cell: not just survival and function, but proliferation as well? Curr Diab Rep 14:492.

Sola D, Rossi L, Schianca GPC, Maffioli P, Bigliocca M, Mella R, Corlianò F, Fra GP, Bartoli E, and Derosa G (2015) Sulfonylureas and their use in clinical practice. Arch Med Sci 11:840-848.

Steneberg P, Rubins N, Bartoov-Shifman R, Walker MD, and Edlund H (2005) The FFA receptor GPR40 links hyperinsulinemia, hepatic steatosis, and impaired glucose homeostasis in mouse. Cell Metab 1:245-258.

Tsujihata Y, Ito R, Suzuki M, Harada A, Negoro N, Yasuma T, Momose Y, and Takeuchi K (2011) TAK-875, an orally available G protein-coupled receptor 40/free fatty acid receptor 1 agonist, enhances glucose-dependent insulin secretion and improves both postprandial and fasting hyperglycemia in type 2 diabetic rats. J Pharmacol Exp Ther 339:228-237.

Wajchenberg BL (2007) Postprandial glycemia and cardiovascular disease in diabetes mellitus. Arq Bras Endocrinol Metabol 51:212-221.

Wang Z and Thurmond DC (2009) Mechanisms of biphasic insulin-granule exocytosis - roles of the cytoskeleton, small GTPases and SNARE proteins. J Cell Sci 122:893-903.

Weir GC and Bonner-Weir S (2004) Five stages of evolving beta-cell dysfunction during progression to diabetes. Diabetes 53 (Suppl 3):S16-S21.

Winzell MS and Ahrén B (2007) G-protein-coupled receptors and islet function-implications for treatment of type 2 diabetes. Pharmacol Ther 116:437-448.

Wolenski FS, Zhu AZX, Johnson M, Yu S, Moriya Y, Ebihara T, Csizmadia V, Grieves J, Paton M, Liao M, et al. (2017) Fasiglifam (TAK-875) alters bile acid homeostasis in rats and dogs: a potential cause of drug induced liver injury. Toxicol Sci 157:50-61.

Address correspondence to: Katarzyna Bazydlo-Guzenda, Research and Development Center, Celon Pharma SA, Marymoncka 15 St., Kazun Nowy, Poland. E-mail: katarzyna.bazydlo@celonpharma.com 